\title{
Rechtliche Grundfragen der EU-Erweiterung
}

\author{
Von Thomas Bruha und Oliver Vogt
}

\section{Einführung}

Die Europäische Union steht vor der größten Erweiterungsrunde ihrer Geschichte. Wenn der Europäische Rat im Dezember 1997 den Empfehlungen der Kommission folgt, so werden Anfang 1998 Beitrittsverhandlungen mit zunächst fünf mittel- und osteuropäischen Staaten (Ungarn, Polen, Tschechische Republik, Estland und Slowenien) sowie mit Zypern aufgenommen werden. Die Tür zum Beitritt soll jedoch auch für diejenigen Länder offen bleiben, in denen der Transformationsprozeß nach Einschätzung der Kommission weniger weit fortgeschritten ist (Rumänien, Bulgarien, Lettland, Litauen, Slowakei). Sie dürfen in ihren Beitrittsbestrebungen weiterhin auf Unterstützung seitens der Europäischen Union rechnen, voraussichtlich sogar in verstärktem Maße (Einbindung dieser Staaten in eine sog. Europakonferenz). ${ }^{1}$ Weitere Beitrittsanträge liegen vor (Türkei, Malta, Schweiz), von denen zur Zeit jedoch nur derjenige der Türkei politisch eingefordert wird. ${ }^{2}$ Darüber hinaus ist nicht auszuschließen, daß nach Abschluß der anstehenden Erweiterung noch weitere Staaten einen Antrag auf EU-Mitgliedschaft stellen werden. Eine rasche Folge mehrerer Erweiterungsrunden und mit ihnen ein Anwachsen der Union auf 20 oder 21, 25 oder 26 (jeweils mit oder ohne Zypern) oder gar bis zu 35 oder mehr Mitglieder liegt daher durchaus im Bereich des Möglichen.

Bereits auf dem Europäischen Gipfel der Staats- und Regierungschefs in Kopenhagen im Juni 1993 war die politische Grundsatzentscheidung zur Osterweiterung getroffen worden. ${ }^{4}$ Seit dem Gipfel von Essen im Dezember 1994 verfolgt die Union dabei eine zweifache

1 Siehe Agenda 2000 - Eine stärkere und erweiterte Union, Beilage 5/97 zum Bulletin der EU, S. $62-65$.

Siehe auch G. Burghardt / F. Cameron, The Next Enlargement of the European Union, European Foreign Affairs Review 2/1997 S. 7-21.

Schlußfolgerungen der Tagung des Europäischen Rates der Staats- und Regierungschefs in Kopenhagen am 21. und 22. Juni 1993, Europa-Archiv 1993, D 258 ff., 263: "Der Europäische Rat hat heute beschlossen, daß die assoziierten mittel- und osteuropäischen Länder, die dies wünschen, Mitglieder der Europäischen Union werden können." 
Vorbereitungsstrategie: ${ }^{5}$ Zum einen unterstützt sie die zehn mittel- und osteuropäischen Länder bei ihrem Transformationsprozeß durch eine "Pre-accession"-Strategie, ${ }^{6}$ die auf den Europaabkommen, dem PHARE-Programm und dem sog. strukturierten Dialog beruht. Zum anderen versucht die Union, sich selbst auf den Beitritt weiterer Mitglieder vorzubereiten, sich "erweiterungsfähig" zu machen. Hierbei muß sie sowohl institutionelle Fragen, wie die der zukünftigen Entscheidungsstrukturen bei einer gewachsenen Mitgliederzahl, als auch materielle Fragen lösen: Es liegt angesichts der (noch) geringen wirtschaftlichen Leistungsfähigkeit der Beitrittskandidaten auf der Hand, daß bisherige Regelungen vor allem in der Strukturpolitik und der Gemeinsamen Landwirtschaftspolitik nicht unverändert beibehalten werden können. $^{7}$

Die institutionellen Reformen sollten nach den Schlußfolgerungen des Essener Gipfels ${ }^{8}$ eigentlich Gegenstand der Regierungskonferenz zur Revision des Maastrichter Vertrages sein. Dort hat man sich bezüglich der für die Erweiterung bedeutsamen Fragen (Stimmengewichtung und Abkehr vom Einstimmigkeitsprinzip im Rat, Zusammensetzung der Kommission) aber nur zum Teil auf Reformen einigen können. Der am 2. Oktober 1997 unterzeichnete Vertrag von Amsterdam ${ }^{9}$ hat diese Reformen überdies mit zahlreichen Kautelen wie Kompensationsvorbehalte, Erzwingung von Einstimmigkeitsentscheidungen unter Berufung auf gewichtige nationale Interessen u.a. versehen. Ohnehin soll die erreichte Reform nur bis zu einer Union von 20 Mitgliedstaaten, also bis zur Aufnahme von maximal fünf neuen Mitgliedern, tragfähig sein; für eine darüber hinausgehende Erweiterung ist bereits eine neue Reformkonferenz ("Amsterdam II") geplant. Wie sich dies mit der oben genannten Empfehlung der Kommission verträgt, die Verhandlungen mit "5 +1" Beitrittskandidaten zu beginnen, mag dahinstehen.

Noch weniger weit gediehen sind die für eine Erweiterung erforderlichen materiellen Reformen. Die besonders heiklen Themen sind bislang nur in dem Kommissionspapier "Agenda 2000" 10 behandelt. Dort werden nicht nur die einzelnen Beitrittskandidaten begutachtet und Empfehlungen abgegeben. Die Kommission legt auch Vorschläge zur Reform der Gemeinsamen Landwirtschaftspolitik, der Strukturpolitiken und zur zukünftigen Finan-

5

6

7

8

9

10

Schlußfolgerungen des Europäischen Rates der Staats- und Regierungschefs in Essen am 9. und 10. Dezember 1994, Internationale Politik 1995 Nr. 1, S. 83 ff., 89.

Der erste Kommissionsentwurf der Heranführungssstrategie findet sich im Anhang IV zu den Schlußfolgerungen des Essener Gipfels, Internationale Politik (oben Fn. 5), S. 98 ff.

Siehe zu Reformbedarf und den verschiedenen Ansätzen R. Hrbek (Hrsg.), Die Reform der Europäischen Union, 1997.

Siehe Schlußfolgerungen (oben Fn. 5), S. 89.

Vertrag von Amsterdam. Text und konsolidierte Fassungen des EU- und EG-Vertrags mit einer Einführung von A. Bardenhewer, Baden-Baden 1997.

Oben Fn. 1. 
zierung der Union vor. ${ }^{11}$ Diese Empfehlungen müssen aber erst noch politisch umgesetzt werden.

Glaubt man den politischen Verlautbarungen nach Abschluß des Amsterdamer Gipfels, so sind dennoch die wesentlichen Vorbereitungen für die erste Runde der Osterweiterung getroffen; die Verhandlungen können beginnen. Dabei handelt es sich in erster Linie um einen politischen Vorgang: Im Vordergrund stehen nicht Rechtsfragen, sondern Fragen des politischen Willens und der wirtschaftlichen Machbarkeit. Veränderungen in der Mitgliedschaft wirken sich auf das ganze Gemeinschaftssystem aus (finanziell, institutionell, dezisionell). Kein Bereich ist ausgeschlossen, da ein Beitritt nur zur Union als ganzer möglich ist. ${ }^{12}$ Damit ist insbesondere auch der politisch besonders sensible Bereich der Gemeinsamen Außen- und Sicherheitspolitik berührt, die "auf längere Sicht" in eine gemeinsame Verteidigungspolitik, "zu gegebener Zeit" auch zu einer gemeinsamen. Verteidigung führen soll. ${ }^{13}$ Die Frage der Neuaufnahme weiterer EU-Mitglieder hat also auch eine stark bündnisbezogene Seite, mag die Parallelmitgliedschaft in WEU und NATO auch nicht Voraussetzung der EU-Mitgliedschaft $\operatorname{sein}^{14}$. Die Rolle, die das Recht angesichts dieses Primats des Politischen spielen kann, ist naturgemäß sehr begrenzt. Das heißt aber nicht, daß die Frage einer Erweiterung oder Nichterweiterung der Europäschen Union allein dem uneingeschränkten politischen Ermessen der beteiligten Staaten unterliegt. Vielmehr lassen sich dem Unions- und Gemeinschaftsrecht ${ }^{15}$ eine Reihe von normativen Vorgaben und rechtlichen Voraussetzungen entnehmen, die es im folgenden auf zuzeigen gilt.

\section{Voraussetzungen der Erweiterung}

\section{Europäischer Staat}

Der Vertrag von Maastricht hat die bisherigen Beitrittsklauseln der drei Gemeinschaftsverträge (Art. 237 EGV, 205 EAGV und 95 EGKSV) durch eine einheitliche Bestimmung

Agenda 2000, S. 97 ff.

Art. O EUV.

13 Siehe Art. J.4 Abs. 1 EUV. Die Neufassung im Amsterdamer Vertrag ist mit Art. 17 (ex-Art. J. 7) bezeichnet und weist einen leicht geänderten Wortlaut auf.

Siehe zur WEU Art. J.4 Abs. 2 EUV. Der Vertrag von Amsterdam geht mit der Verknüpfung von EU und WEU noch einen Schritt weiter: Künftig soll die WEU mit den sog. Petersberg-Aufgaben, also humanitären und friedenserhaltenden Aufgaben, aber auch mit Kampfeinsätzen betraut werden, Art. 17 (ex-Art J.7) Abs. 2 EUV.

Siehe zur Abgrenzung von Unionsrecht und Gemeinschaftsrecht C. Koenig / M. Pechstein, Die Europäische Union, 1995, S. 3 ff. 
über den Beitritt zur Union ersetzt. Sie enthält neben Verfahrensvorschriften allein die Regelung, daß nur europäische Staaten Anträge auf Unionsmitgliedschaft stellen können:

\section{Art. O EUV (Auf nahme weiterer Mitglieder)}

Jeder europäische Staat kann beantragen, Mitglied der Union zu werden. Er richtet seinen Antrag an den Rat; dieser beschließt einstimmig nach Anhörung der Kommission und nach Zustimmung des Europäischen Parlaments, das mit absoluter Mehrheit seiner Mitglieder beschließt.

Die Aufnahmebedingungen und die durch eine Aufnahme erforderlichen Anpassungen der Verträge, auf denen die Union beruht, werden durch ein Abkommen zwischen den Mitgliedstaaten und dem antragstellenden Staat geregelt. Das Abkommen bedarf der Ratifikation durch alle Vertragsstaaten gemäß ihren verfassungsrechtlichen Vorschriften.

Das wirft die Frage auf, was unter "europäisch" zu verstehen ist: Ist die Lage des Staatsgebietes entscheidend oder kommt es statt dessen (oder) auch auf das historisch-kulturell geprägte Zugehörigkeitsgefühl der Bevölkerung zu Europa an? Oder, dritte Möglichkeit, handelt es sich bei dem Attribut "europäisch" um einen "beliebigen Inklusions- bzw. Exklusionsbegriff", ${ }^{16}$ der ausschließlich im politischen Prozeß zu konkretisieren ist? Von diesen drei Deutungsvarianten scheidet allein die letztgenannte aus. Das Antragserfordernis des "europäischen Staates" steht nun einmal im Vertrag, so daß ihm die normative Bedeutung nicht ohne weiteres abgesprochen werden kann.

\section{a) Geographischer Europabegriff}

Im Unterschied zu anderen Kontinenten weist Europa nicht nach allen Richtungen hin "natürliche" Grenzen auf; aus geographischer Sicht gehört es vielmehr zum Eurasischen Kontinent. Allein geschichtliche, wirtschaftliche und kulturelle Gründe rechtfertigen es, Europa als selbständigen Erdteil aufzufassen. Insbesondere die Ostgrenze Europas wanderte jahrhundertelang je nach den philosophischen, geschichtlichen, religiösen, politischen oder wirtschaftlichen Auffassungen. ${ }^{17}$ Diese Grenze erweist sich daher eher als Akt menschlicher Willkür denn als Anerkennung natürlicher Gegebenheiten. ${ }^{18}$ In neuerer Zeit gilt als Grenze zu Asien eine gedachte Linie aus Uralgebirge, Uralfluß, Kaspischem Meer und Manytsch-Niederung sowie der Folge aus Asowschem Meer, Schwarzem Meer, Bospo-

H. Münkler: Die politische Idee Europa; in: Delgado / Lutz-Bachmann (Hrsg.), Herausforderung Europa, 1995, S. 9 ff., 10.

17

Münkler a.a.O., S. $11 \mathrm{f}$.

18 J. Isensee, Nachwort in: Isensee (Hrsg.) Europa als politische Idee und als rechtliche Form, 1993, S. $103 \mathrm{ff} ., 106$. 
rus, Marmara-Meer und Dardanellen. ${ }^{19}$ Faßt man den Begriff "europäischer Staat" also rein geographisch auf, so müßte diese Grenzziehung, die zur Zeit der Abfassung der Römischen Verträge vorherrschend war, wohl auch heute zugrunde gelegt werden.

\section{b) Historisch-kultureller Europabegriff}

Sind schon die geographischen Kriterien zumindest zweifelhaft, so verschwimmen die Konturen einer historisch-kulturellen Definition vollends. ${ }^{20}$ Blickt man in die Antike, so gehört Kleinasien sicherlich zur Wiege europäischer Kultur, wogegen der größte Teil des heutigen Nord- und Westeuropa damals so gut wie unbekannt war. Ähnliches gilt für den Mittelmeerraum; alle Anrainerstaaten des mare nostrum, also auch die nordafrikanischen, gehörten zu einem einheitlichen Kulturraum. Die mittelalterlichen Anfänge Europas als politisch-kultureller Größe dagegen werden oft auf die karolingische Reichsgründung datiert, mit der sich auch eine Abtrennung vom Mittelmeerraum vollzog. Hinzukam die theologisch-dogmatische Trennung von Kleinasien im Schisma von Byzanz. Bestimmend für die politische und gesellschaftliche Identität Europas wurde daher nicht allein die mittelalterliche "Einheit des Christentums", sondern ebenso die sich herausbildende Polarität von Kaiser und Papst, Reich und Kirche, die im Gegensatz zum Cäsaropapismus der Reiche von Byzanz und Moskau stehen. Faßt man den Begriff Europa dagegen eher (macht-) historisch auf, so muß man auch Rußland als einen seit der Neuzeit nicht mehr wegzudenkenden Faktor einbeziehen. Eine Grenzziehung allein nach religiösen Merkmalen wiederum, die ebenso kulturprägend sind wie die machtpolitischen und staatstheoretischen, würde aber nicht nur das arabisch-muslimische kulturelle Erbe Europas ignorieren, sondern auch mit der säkularen Ausrichtung moderner Staaten in Konflikt treten. Geographischer und kultureller Europabegriff sind also offensichtlich nicht deckungsgleich; über die hieraus gezogene Schlußfolgerung: "Was Europa ist, bestimmt von jeher allein der Europäer", ${ }^{21}$ wird man allerdings streiten können.

\section{c) Stellungnahme}

Rechtlich betrachtet, darf die Schwierigkeit einer exakten Grenzziehung jedenfalls nicht zu einer allzu restriktiven Auffassung führen. Vielmehr legen die Geschichte des europäischen Integrationsprozesses und die vertraglichen Zielbestimmungen ein sehr weites Europaverständnis nahe. Nach der Präambel des EGV ist die Gemeinschaft auf einen "immer engeren

19

20

21

Der Große Brockhaus, 16. Auflage 1953, Stichwort Europa.

Siehe zum folgenden neben Münkler und Isensee (op. cit.) E. Tortarolo, Europa. Zur Geschichte eines umstrittenen Begriffs, in: A. von Bogdandy (Hrsg.), Die Europäische Option, 1993, S. 21 ff.

Isensee (oben Fn. 18), S. $112 \mathrm{f}$. 
Zusammenschluß der europäischen Völker" (Abs. 1) angelegt. Der Prozeß der wirtschaftlichen Integration soll Frieden und Freiheit wahren und wird verbunden mit der "Aufforderung an die anderen Völker Europas, die sich zu den gleichen hohen Zielen bekennen, sich diesen Bestrebungen anzuschließen" (Abs. 8). Eine noch deutlichere Sprache findet die nach dem Fall des Eisernen Vorhangs formulierte Präambel des Unionsvertrages, in deren zweiten Absatz die Vertragsparteien an die "historische Bedeutung der Überwindung der Teilung des europäischen Kontinents" erinnern und sich die Aufgabe stellen, "feste Grundlagen für die Gestalt des zukünftigen Europas zu schaffen". ${ }^{22}$ Dies rechtfertigt es, mit der Bestimmung des Begriffs "europäischer Staat" bis an die Grenze der möglichen Bedeutung zu gehen.

Deshalb gilt: Nur Staaten, die Europa nach keinem der zuvor genannten Kriterien zugeordnet werden können, dürfen nach geltender Rechtslage nicht in die Gemeinschaft aufgenommen werden. Betroffen davon sind etwa die fünf asiatischen Mitgliedstaaten der GUS (Kasachstan, Kirgisistan, Tadschikistan, Turkmenistan und Usbekistan), aber auch die ehemaligen französischen Kolonien in Nordafrika. ${ }^{23}$ Umgekehrt darf kein Staat, der anhand der genannten Kriterien als "europäisch" bezeichnet werden kann, von vornherein aus dem Kreis potentieller Beitrittskandidaten ausgegrenzt werden. Als Beispiele seien die "rein europäischen" Nachfolgerepubliken der GUS, also Weißrußland, die Ukraine und die Republik Moldau genannt.

Deutungsspielraum, der integrationspolitisch ausgefüllt werden kann, besteht also nur bei den "Grenzfällen". Rußland und die Türkei sind die wichtigsten Beispiele: Beide Staaten liegen mit dem weitaus größeren Teil ihres Territoriums in Asien. Zumindest im Falle Rußlands kann der Bevölkerung die "europäische Orientierung" jedoch kaum abgesprochen werden, allein schon deswegen, weil nur ein geringer Prozentsatz der Bevölkerung in den dünn besiedelten asiatischen Republiken östlich des Urals wohnt. Eine strenge Bindung an die "geographischen" Grenzen Europas würde willkürlich wirken und wird auch den Zielen der Union nicht gerecht. Auch die bisherigen Mitgliedstaaten haben in beschränktem Umfang außereuropäische Teile ihres Hoheitsgebietes in den Geltungsbereich der Verträge mit einbezogen, wenn auch aus anderen Gründen. ${ }^{24}$ Die Frage der Einordnung Rußlands ist auch nicht nur theoretischer Natur: Erst kürzlich hat der russische Premier Tschernomyrdin das Interesse seines Landes an einer Mitgliedschaft bekundet.

Schwieriger mag die Einschätzung bei der Türkei sein, deren europäischer Teil vergleichsweise klein ist (obgleich immerhin der historische Stadtkern Istanbuls auf der europäischen

22

23

24

Präambel EUV, Abs. 1 und 2.

Siehe Anwort der Kommission an Marokko, EA 1987, Z 207.

W. Meng in: v.d. Groeben/Thiesing/Ehlermann, Kommentar zum EWG-Vertrag, 4. Aufl. 1991, Art. 237 EGV, Rn. 32. 
Seite liegt). Hält man die soziokulturelle Verfassung für das entscheidende Merkmal, so ist zu bedenken, daß die offizielle Abkehr von islamischen Traditionen durch die Reformen Atatürks erst relativ neueren Datums ist und vor allem in den ländlichen Gebieten unvollständig geblieben ist. Zudem erstarken in der Türkei zunehmend Kräfte, die die osmanische Vergangenheit erneuern wollen und die Einheit der islamischen Welt beschwören. Nur eine relativ kleine Oberschicht ist "westlich" orientiert. ${ }^{25}$ Dennoch dürfte die Frage der Zugehörigkeit der Türkei zu Europa längst im positiven Sinne beantwortet sein. Schon 1964 ist der Türkei die Mitgliedschaft in der damaligen EWG ausdrücklich in Aussicht gestellt wor ${ }^{-}$ $\operatorname{den}^{26}$. Von dieser rechtlichen Zielsetzung sind nie Abstriche gemacht worden, auch nicht bei Errichtung der Zollunion im Jahr 1996. ${ }^{27}$ Die politische Entscheidung der Gemeinschaft zugunsten der Zugehörigkeit der Türkei zu Europa ist also schon gefallen. ${ }^{28}$

Überdies gehören beide Länder dem Europarat an, der ebenfalls nur "europäischen Staaten" offensteht. ${ }^{29} \mathrm{Zu}$ der Frage, ob die ehemaligen Republiken der Sowjetunion "europäisch" im Sinne der Europarats-Satzung sind, hat die Parlamentarische Versammlung bereits 1992 Stellung genommen. ${ }^{30}$ Danach war die Zugehörigkeit Rußlands zu Europa ebenso selbstverständlich ("obvious" im englischen Originaltext) wie die von Weißrußland und der Ukraine. Es kann angenommen werden, daß die nach dem Europarat gegründeten Europäischen Gemeinschaften denselben "Europabegriff" verwenden wollten. Dies gilt um so mehr, als beide Organisationen, etwa auf dem Gebiet des Menschenrechtsschutzes, zunehmend miteinander verflochten sind und koordinierte Maßnahmen zum Schutze der "europäischen Identität" entwickeln (insbesondere im Kulturbereich, vgl. Art. 128 Abs. 3 EGV). Sicherlich wird man dem Europarat insoweit keine "allgemeinverbindliche" Definitionskompetenz zugestehen können, die auch den Europabegriff des Art. O EUV präjudiziert. Das verbietet sich schon wegen der unterschiedlichen Zustimmungserfordernisse: Art. O EUV setzt Einstimmigkeit aller Mitgliedstaaten und die Zustimmung des Europäischen Parlamentes voraus. Nach Art. 4 i.V. m. Art. 20 lit. c) der Europarats-Satzung genügt hingegen eine Zweidrittelmehrheit der Mitgliedstaaten. Sofern die Aufnahmepraxis des Euro-

Vgl. H. Bacia: Mehr Realismus in der Türkei, F.A.Z. Nr. 227 v. 30. Sept. 1997.

Absatz 3 der Präambel sowie Art. 28 des Assoziationsabkommens, ABl. 1964, Nr. 217, S. 3685 ff. Beschluß 1/95 des Assoziationsrates vom 22.12.1995 zur Errichtung der Zollunion, ABl. L 35 v. 13.2.1996, S.1, verweist auf die Ziele des Assoziationsabkommens von 1964, insbes. dessen Art. 28.

Ähnlich E. Klein, in: Hailbronner u.a. (Hrsg.), Handkommentar zum EU-Vertrag, Loseblatt, 3. Lieferung August 1994, Art. O, Rn. 8; R. Geiger, EG-Vertrag, 2. Aufl. 1995, Art. O Rn. 2.

Art. 4 S.1 der Satzung des Europarates lautet wie folgt: "Jeder europäische Staat, der für fähig und gewillt befunden wird, die Bestimmungen des Artikels 3 zu erfüllen, kann vom Ministerkomittee eingeladen werden, Mitglied des Europarates zu werden."

Bericht der Parlamentarischen Versammlung, The Geographical Enlargement of the Council of Europe, HRLR Vol. 13 (1992), No. 5-6, S. 230 ff. 
parates aber von den Mitgliedstaaten der EU und dem Europäischen Parlament mitgetragen wird (was bei letzterem nur im politischen Sinne gemeint sein kann), dürfte die Frage, ob ein Staat "europäisch" im Sinne der Europarats-Satzung ist, auch für Art. O EUV politisch mitentschieden sein.

Dies könnte in Zukunft vielleicht für die drei transkaukasischen Nachfolgestaaten der UdSSR (Armenien, Aserbaidschan und Georgien) von Bedeutung werden, deren Auf nahme in den Europarat immerhin in Erwägung gezogen worden ist, ${ }^{31}$ obwohl sie außerhalb der oben skizzierten "geographischen" Grenzen Europas liegen. Interessant ist in diesem Zusammenhang ferner, daß auch die Parlamentarische Versammlung des Europarates vor einer abschließenden Entscheidung - also auch vor einer Ausgrenzung - der kaukasischen Republiken zurückgeschreckt ist. In salomonischer Weise macht sie deren Beitrittsperspektive vielmehr davon abhängig, ob sie selbst ihren Willen klar zum Ausdruck bringen, als Teil Europas anerkannt zu werden. ${ }^{32}$ Ob damit eine Offerte an alle zwölf GUS-Staaten verbunden sein soll, ist zur Zeit wohl noch offen. Im organisatorischen Rahmen der UNO gehören diese Staaten übrigens alle der Gruppe der "Europäischen Staaten" an. Entsprechend werden sie etwa auch von den Aktivitäten der UN Economic Commission for Europe (ECE) erfaßt.

\section{Kriterien des Kopenhagener Gipfels}

Angesichts des hier vertretenen weiten Europaverständnisses kommt den ungeschriebenen materiellen Beitrittsvoraussetzungen die entscheidende Bedeutung zu. Obwohl sie im Unionsvertrag zur Zeit noch nicht ausdrücklich verankert sind, besteht über ihre Existenz dem Grunde nach Einvernehmen. ${ }^{33}$ Sie lassen sich mit den Begriffen Verfassungsstaatlichkeit, Binnenmarktfähigkeit, Integrationswilligkeit (der Beitrittskandidaten) und Aufnahmebzw. Erweiterungsfähigkeit (der Gemeinschaft) umreißen. Konkreter sind diese vier Voraussetzungen in den Schlußfolgerungen des Europäischen Rates von Kopenhagen (Juni 1993) niedergelegt: ${ }^{34}$

Wegen der europäischen Orientierung ihrer Bevölkerung siehe den zitierten Bericht der Parlamentarischen Versammlung, HRLR, a.a.O., S. $230 \mathrm{ff}$.

Siehe Bericht der Parlamentarischen Versammlung, HRLR, a.a.O. S. 231 und 233.

Siehe M. Heintzen, Gemeineuropäisches Verfassungsrecht in der Europäischen Union, EuR 1997, S. 1 ff., 6.; R. Geiger, EG-Vertrag, 2. Aufl. 1995, Art. O Rn.3; E. Klein (oben Fn. 28), Rn. 9 ff.; W. Meng (oben Fn. 24), Rn. 40 ff.; C. Vedder in: Grabitz/Hilf, Kommentar zur Europäischen Union, Loseblatt (Oktober 1996), Art. 237 Rn. 5-8.

Siehe EA 1993, D. 264. 


\section{Kopenhagener Kriterien für eine Erweiterung der Union}

[1.] Als Voraussetzung für die Mitgliedschaft muß der Beitrittskandidat eine institutionelle Stabilität als Garantie für demokratische und rechtsstaatliche Ordnung, für die Wahrung der Menschenrechte und den Schutz von Minderheiten verwirklicht haben;

[2.] [die Mitgliedschaft] erfordert ferner eine funktionsfähige Marktwirtschaft sowie die Fähigkeit, dem Wettbewerbsdruck und den Marktkräften innerhalb der Union standzuhalten.

[3.] Die Mitgliedschaft setzt ferner voraus, daß die einzelnen Beitrittskandidaten die aus einer Mitgliedschaft erwachsenden Verpflichtungen übernehmen und sich die Ziele der politischen Union sowie der Wirtschafts- und Währungsunion zu eigen machen können.

[4.] Die Fähigkeit der Union, neue Mitglieder aufzunehmen, dabei jedoch die Stoßkraft der europäischen Integration zu erhalten, stellt ebenfalls einen sowohl für die Union als auch für die Beitrittskandidaten wichtigen Gesichtspunkt dar.

\section{a) Rechtsnatur und Verbindlichkeit der "Schlußfolgerungen"}

Die Rechtsnatur derartiger "Schlußfolgerungen" ist noch nicht abschließend geklärt. Fest steht allerdings, daß der Europäische Rat kein verbindliches Gemeinschaftsrecht schaffen kann, denn er ist weder ein Organ der Gemeinschaft, noch handelt er im Rahmen der gemeinschaftlichen Rechtsetzungsverfahren. ${ }^{35}$ Unbeschadet seiner ihm nach Art. D des Unionsvertrages zukommenden politischen Führungsaufgabe kann er wohl auch nicht als Organ der Union qualifiziert werden, denn diese verfügt nach h.M. nicht über eine eigene Rechtspersönlichkeit. ${ }^{36}$ Nach einer in der Literatur vertretenen Ansicht handelt es sich beim Europäischen Rat um eine "institutionalisierte, periodisch stattfindende zwischenstaatliche Konferenz", deren Themen durch den Unionsvertrag festgelegt sind. Seine Beschlüsse seien daher auch nicht gemeinschaftsrechtlicher, sondern völkerrechtlicher Natur. Sie könnten im Einzelfall als völkerrechtliche Vereinbarungen gedeutet werden, die allerdings gegebenenfalls durch die nationalen Parlamente ratifiziert werden müßten. ${ }^{37}$ Letztlich kann diese Frage hier jedoch offenbleiben, denn die Kopenhagener Kriterien geben nach

Vgl. A. Bleckmann, Europarecht, 6. Aufl. 1997, Rn. 65; C. Koenig / M. Pechstein, Die Europäische Union, 1995, S. 69 ff.; J. P. Jaqué, in: v.d. Groeben/Thiesing/Ehlermann (Hrsg.), Kommentar zum EU-/EG-Vertrag, 5. Aufl. 1997, Art. D Rn. 17; M. Hilf/E. Pache in: Grabitz/Hilf (Hrsg.), Kommentar zur Europäischen Union, Loseblatt, 8. Lieferung Mai 1995, Art. D Rn. 8, 32, 37 ff.

37 Str., vgl. Bleckmann, a.a.O. Rn. 70, Hilf/Pache, a.a.O., Rn. 8, m. w. Nachw.

Siehe Bleckmann, a.a.O., Rn. 70. Diese Auffassung scheint auch das BVerfG in seinem Maastricht-Urteil zugrundezulegen, vgl. BVerfGE 89, S. 155 ff., 175 ff.; Hilf/Pache, a.a.O., Rn. 41, sprechen dagegen von einem Vertragsorgan der Mitgliedstaaten im Rahmen des EUV. 
der hier vertretenen Auffassung geltendes Recht wieder. Alle genannten Erfordernisse lassen sich aus den politischen und wirtschaftlichen Zielbestimmungen und Prinzipien der Gemeinschaftsverträge und des Unionsvertrages herleiten: ${ }^{38}$

\section{b) Verfassungsstaatlichkeit (1. Kriterium)}

Als Rechtsgemeinschaft kann die Europäische Union nur funktionsfähig sein, wenn ihre Mitgliedstaaten selbst die grundlegenden Strukturprinzipien der Demokratie und Rechtsstaatlichkeit beachten. ${ }^{39}$ Hierauf verweist Art. F Abs. 1 EUV mit seiner schlichten Feststellung, daß die Regierungssysteme der Mitgliedstaaten auf demokratischen Grundsätzen beruhen. Nach Art. F Abs. 2 EUV achtet die Union die Menschenrechte und Grundfreiheiten als allgemeine Grundsätze des Gemeinschaftsrechts. Als Rechtsquelle für diese Freiheiten verweist die Vorschrift nicht nur auf die Europäische Menschenrechtskonvention, sondern auch auf die "gemeinsamen Verfassungsüberlieferungen der Mitgliedstaaten". Das Erfordernis einer stabilen demokratischen Ordnung folgt aber auch aus "operationellen" Bestimmungen wie z.B. Art. 8 b EGV, der den Unionbürgern an jedem Wohnsitz innerhalb der Union das aktive und passive Wahlrecht bei Kommunalwahlen und bei den Wahlen zum Europäischen Parlament einräumt. Ein solches Wahlrecht kann nur dann im vollen Umfang garantiert sein, wenn ein Mitgliedstaat auch innerstaatlich die u.a. im Direktwahlbeschluß von 1976 niedergelegten Wahlrechtsgrundsätze beachtet. Ähnlich ist das Erfordernis der Rechtsstaatlichkeit unverzichtbare conditio der EU-Mitgliedschaft. So baut etwa das gesamte Binnenmarktrecht auf den Prinzipien rechtsstaatlich korrekter Implementation (Prinzipien "guter Verwaltungsführung") und effektiven Rechtsschutzes auf. Bei dem Kriterium der Verfassungsstaatlichkeit handelt es sich daher durchaus um ein rechtliches und nicht nur um ein politisches Kriterium. ${ }^{40}$

Vor diesem Hintergrund erscheint es nur konsequent, daß der Vertrag von Amsterdam die unter Punkt 1 der Kopenhagener Kriterien genannten Anforderungen mit Ausnahme des Minderheitenschutzes jetzt auch ausdrücklich im Rahmen der Neufassung des Artikel O EUV verankert hat. Die Bestimmung wird im ersten Satz ergänzt und in der konsolidierten Fassung in Art. 49 EUV umbenannt:

A.A. C. Vedder zum wortlautgleichen Art. 237 EWGV, in: Grabitz/Hilf (Fn. 33), Rn. 5, dem zufolge die obengenannten ungeschriebenen Beitrittsvoraussetzungen politischer und wirtschaftlicher Natur sind und damit nicht zu einer rechtlichen Ausgrenzung beitrittswilliger Staaten führen könnten. Wie hier u.a. G. Meier, Rechtliche Grenzen für einen Beitritt zu den Europäischen Gemeinschaften, EuR 1978, S. 12 ff, 16 ff.

Der hier vertretenen Auffassung steht die Einteilung der Beitrittskriterien in "politische" und "wirtschaftliche" Kriterien, wie sie die Kommission in ihrer Agenda 2000, S. 44 ff. vornimmt, nicht entgegen. 


\section{Art. 49 (ex-Art. O) EUV}

Jeder europäische Staat, der die in Artikel 6 Absatz 1 genannten Grundsätze achtet, kann beantragen, Mitglied der Union zu werden. (übriger Text unverändert).

Die Verweisung bezieht sich auf die Neufassung des früheren Artikel F, der in Zukunft wie folgt lauten wird:

\section{Art. 6 (ex-Art. F) EUV}

(1) Die Union beruht auf den Grundsätzen der Freiheit, der Demokratie, der Achtung der Menschenrechte und Grundfreiheiten sowie der Rechtsstaatlichkeit; diese Grundsätze sind allen Mitgliedstaaten gemeinsam.

Wie aus dem zuvor Gesagten deutlich wird, handelt es sich bei diesen Ergänzungen letztlich nur um eine deklaratorische Bestätigung bereits geltenden Rechts. Wenn diese Fassung, wie vorgesehen, noch vor dem Beitritt neuer Mitgliedstaaten in Kraft tritt, so wird eine ausdrückliche, geschriebene Beitrittsvoraussetzung vorliegen. An der rechtlichen Qualität dieses Erfordernisses dürften dann keine Zweifel mehr bestehen.

\section{c) Binnenmarktfähigkeit (2. Kriterium)}

Im Unterschied zum Erfordernis der Verfassungsstaatlichkeit der Beitrittskandidaten werden die in Punkt 2 der Kopenhagener Kriterien genannten Anforderungen an ihre Wirtschaftsverfassung und Wettbewerbsfähigkeit auch durch den Amsterdamer Vertrag nicht ausdrücklich im Unionsrecht verankert. Gleichwohl lassen sich auch diese Voraussetzungen für einen Beitritt aus dem geltenden Gemeinschaftsrecht ableiten. ${ }^{41}$ Zwei Ebenen lassen sich unterscheiden: Zum einen setzt eine funktionsfähige Marktwirtschaft gewisse rechtliche Grundbedingungen voraus. Hierzu gehören vor allem so grundlegende Garantien wie Eigentum, Gewerbefreiheit, effektiver Wettbewerbsschutz u.a.. Die Berechtigung dieser Anforderung an beitrittswillige Länder ergibt sich daraus, daß der Gemeinsame Markt, wiewohl eigentumsrechtlich neutral (siehe Art. 222 EGV), auf einer vom Grundsatz her marktwirtschaftlich und am Wettbewerb ausgerichteten Wirtschaftsverfassung seiner Mitgliedstaaten basiert. ${ }^{42}$ Wenn auch keine allzu hohen Anforderungen an die Homogenität der Wirtschaftsordnungen gestellt werden dürfen, ${ }^{43}$ so setzt das Funktionieren des Binnenmarktes doch eine ähnliche Ausrichtung aller Mitgliedstaaten voraus. Aber auch das Erfor-

41

Siehe Klein (oben Fn. 28), Rn. 10; W. Meng (oben Fn. 24), Art. 237 EGV, Rn. 45; Heintzen (oben Fn. 33), S. 7.

42

43

Art. 3 lit g EGV, siehe zur Wirtschaftsverfassung des Binnenmarktes: G. Nicolaysen, Europarecht II - Das Wirtschaftsrecht im Binnenmarkt, 1996, S. 317 ff.; P. Behrens, Die Wirtschaftsverfassung der Europäischen Gemeinschaft, in: G. Brüggemeier, Verfassungen für ein ziviles Europa, 1993, S. 73 ff.;

Vedder, a.a.O., Rn. 8. 
dernis der Wettbewerbsfähigkeit ist als rechtliche Beitrittsvoraussetzung zu begründen. Befindet sich die wirtschaftliche Leistungsfähigkeit einer Volkswirtschaft auf einem so niedrigen Niveau, daß ein Beitritt die wirtschaftlichen und sozialen Gemeinschaftsziele aus Art. 2 und der Präambel des EGV gefährdete, so wäre er nicht nur für beide Seiten wirtschaftlich wie politisch inopportun. Er müßte auch aus gemeinschaftsrechtlichen Gründen ausscheiden.

Eine andere Frage sind die Kritierien, anhand derer die Binnenmarktfähigkeit festzustellen ist. Sie sind eher ökonomischer als rechtlicher Natur. Lassen sich die benannten Grundbedingungen einer marktwirtschaftlichen Wirtschaftsordnung noch anhand rechtlicher Kriterien klären, so unterliegt das Merkmal der Wettbewerbsfähigkeit einer im wesentlichen wirtschaftlichen Einschätzung, die nur schwer an spezifisch rechtlichen Kriterien festzumachen ist. Hinzukommt, daß die Union zum einen im Rahmen ihrer Heranführungsstrategie Starthilfe für die Beitrittskandidaten leistet, zum anderen einen Beitritt durch zeitlich befristete Übergangsregimes erleichtern kann. Das schließt es zwar nicht aus, in der Binnenmarktreife eine (zumindest auch) rechtliche Voraussetzung eines Beitritts zu sehen. ${ }^{44}$ Jedoch muß den Mitgliedstaaten und den an der Entscheidung beteiligten Gemeinschaftsorganen hier ein wesentlich weiterer Beurteilungsspielraum zugebilligt werden als etwa bei der Frage der Verfassungsstaatlichkeit. Die rechtliche Grenze für die Aufnahme weiterer Mitgliedstaaten wäre nur in dem Extremfall erreicht, wenn ein Beitrittskandidat wegen seiner unzureichenden rechtlichen und wirtschaftlichen Verfassung auch bei Ausschöpfung aller zulässigen Übergangsregimes und Anschubhilfen durch die Gemeinschaft nicht binnenmarktreif werden kann.

\section{d) Integrationswilligkeit (3. Kriterium)}

Der Beitritt zur Union erfordert ferner die Fähigkeit zur Übernahme der aus der Mitgliedschaft erwachsenden Verpflichtungen. Dazu gehört nicht nur der bereits erreichte acquis der Union (in allen drei Pfeilern) im eigentlichen Sinne. Die Verpflichtung rrstreckt sich auch auf die zum Beitrittszeitpunkt vereinbarten Zielvorgaben (acquis communautaire im weiteren Sinne), mögen diese konkret vorgezeichnet (3. Stufe der Wirtschafts- und Währungsunion) oder inhaltlich wie zeitlich offengehalten sein (Politische Union). Allerdings verlangt das Gemeinschaftsrecht nicht, daß im Zeitpunkt des Beitritts dieser acquis vollständig zu übernehmen ist. Er muß nur übernommen werden können ("ability to take on the obligations of membership"), wie die Kopenhagener Kriterien dies auch deutlich zum Ausdruck bringen. Entsprechend haben alle Beitrittsverträge bislang Übergangsfristen vorgese-

A.A. Vedder (oben Fn. 33), Rn. 5, der das Erfordernis der Binnenmarktreife als Vorwirkung des mit dem Beitritt verbundenen und erst am Ende der Übergangszeit zu erreichenden rechtlichen, wirtschaftlichen und politischen Integrationsstandes auffaßt. 
hen, um die Volkswirtschaften der "Alt-" und "Neumitglieder" allmählich aufeinander abzustimmen. Zum Zeitpunkt des Beitritts muß jedoch gewährleistet sein, daß die "Neumitglieder" gewissermaßen von ihren "Anlagen" her (rechtliche und administrative Strukturen, ökonomische Leistungsfähigkeit) in der Lage sind, alle Verpflichtungen zu übernehmen und daß sie auch den dazu erforderlichen politischen Willen erkennen lassen.

Keine Beitrittsvoraussetzung ist daher insbesondere die Fähigkeit, sofort an der dritten Stufe der Wirtschafts- und Währungsunion teilnehmen zu können. Es genügt die Teilnahme an der zweiten Stufe. ${ }^{45}$ Allerdings können sich "Neumitglieder" nicht auf die für einige "Altmitglieder" vereinbarten Sonderregelungen berufen (Sonderrechte Großbritanniens und Dänemarks bezüglich der Entscheidungen über den Eintritt in die dritte Stufe der Wirtschafts- und Währungsunion). Insofern wird von ihnen mehr verlangt, als von einigen "Altmitgliedern". Das gilt gleichermaßen für die in Amsterdam vereinbarten Sonderrechte bezüglich der Vergemeinschaftung des "Schengen-acquis" ${ }^{46}$ und des Inhalts des Sozialprotokolls. ${ }^{47}$ Auch hier sind einigen Mitgliedstaaten (Großbritannien, Irland und Dänemark) unterschiedlich weit reichende Sonderrechte eingeräumt worden. Diese Pflicht der "Neumitglieder" zur uneingeschränkten Übernahme des acquis communautaire schließt aber nicht aus, daß dieser ihnen gegenüber während der Laufzeit der Übergangsfristen teilweise nicht zur Anwendung kommt (z.B. vorübergehende Beibehaltung von Grenzkontrollen im Personenverkehr trotz voller Verpflichtung der "Neumitglieder" auf den "Schengen-acquis").

\section{e) Erweiterungsfähigkeit der Union (4. Kriterium)}

Bei all diesen Problemen, die sich auf der Seite der Beitrittskandidaten stellen und in concreto noch stellen mögen, ist das vierte Erfordernis der Kopenhagener Kriterien, die Erweiterungsfähigkeit der Europäischen Union, nicht zu gering einzuschätzen. Es ist ein viel beklagtes Faktum, daß die Gemeinschaft trotz der Vervielfachung ihrer bisherigen Mitgliederzahl die für eine Gemeinschaft von sechs Staaten konzipierten Strukturen dem Grunde nach noch nicht überwunden hat. Mit den jetzt anstehenden Erweiterungen nähert sie sich gefährlich einer Situation, in der ein prinzipielles Festhalten am "Grunddesign" der Organisation Effizienz und Legitimation der gemeinschaftlichen Entscheidungsprozesse ernsthaft in Frage stellen könnte. Nicht zuletzt deshalb handelt es sich bei dem Erfordernis der Aufnahme- bzw. Erweiterungsfähigkeit um die vielleicht "politischste" Voraussetzung der Erweiterung. Zum einen ist die Vermittlung zwischen den gleichermaßen aufgegebenen

45

46

47

Siehe Agenda 2000 (oben Fn. 1), S. 49.

Siehe neuer Titel IV (ex-Titel III a) EGV: Visa, Asyl, Einwanderung und andere Poltiken betreffend den freien Personenverkehr.

Siehe Art. 136-143 (ex-Art. 117-120) EGV. 
Zielen der materiellen Aufgabenerfüllung der Gemeinschaft und der Vertiefung des Integrationsprozesses auf der einen Seite, und der Erweiterung der Union auf der anderen Seite, politisch zu treffen. Zum anderen handelt es sich hierbei weitgehend auch um Prognoseentscheidungen, die sich rechtlicher Regelung und Kontrolle weitgehend entziehen. Selbst wenn man dem Recht einen verbindlichen Entscheidungsrahmen entnehmen würde, der politisch nicht überschritten werden darf (Gefährdung der "Stoßkraft der europäischen Integration" - 4. Kopenhagener Kriterium), wäre damit im praktischen Ergebnis also nicht viel gewonnen. Die Frage der Erweiterungsfähigkeit der Union ist primär eine rechtspolitische Frage. Aus diesem Grunde erscheint es auch konsequent, wenn der Europäische Rat in Kopenhagen insoweit nur von einem "wichtigen Gesichtspunkt" - und man sollte hinzufügen: einem überragend wichtigen Gesichtspunkt - gesprochen hat. Rechtlich greifbar wird die Problematik erst dann, wenn es infolge einer Erweiterung zu Systemstörungen kommt. Diese können im Vorfeld der Erweiterung aber kaum antizipiert werden.

\section{Justiziabilität}

Die damit angesprochene Frage der Justiziabilität der Erweiterungsvoraussetzungen ${ }^{48}$ ist daher differenzierend zu beantworten. Wie aus den Ausführungen zu den einzelnen Kriterien ersichtlich, eignen sich nicht alle Voraussetzungen gleichermaßen für die gerichtliche Kontrolle. Schwierig ist auch die vorrangige Frage, ob und auf welche Weise der Europäische Gerichtshof überhaupt mit der Frage der Rechtmäßigkeit einer Erweiterung der Gemeinschaft befaßt werden könnte. Denn die Beitrittsakte als solche sind völkerrechtliche Verträge zwischen den Mitgliedstaaten und den Beitrittskandidaten, Art. O Abs. 2 EUV. Als vertragsänderndes Primärrecht stehen sie im Rang den Gemeinschaftsverträgen und dem Unionsvertrag grundsätzlich gleich. Eine Überprüfungskompetenz des Gerichtshofs, der seine Zuständigkeiten nur aus eben diesem Primärrecht ableiten kann, ist daher nicht ohne weiteres begründbar. Das Gutachtenverfahren des Art. 228 Abs. 6 EGV ist schon deshalb nicht anwendbar, weil es völkerrechtliche Verträge der Gemeinschaft mit dritten Staaten oder Organisationen betrifft (Art. 228 Abs. 1 EGV). Wie aus Art. O Abs. 2 EUV folgt, ist die Gemeinschaft selbst nicht Vertragspartei der Beitrittsverträge.

Denkbar erscheint aber eine Anknüpfung an die unterhalb der Schwelle des Primärrechts stehenden Handlungen der Gemeinschaftsorgane im Zusammenhang mit einem Beitritt. Die Aufnahme neuer Mitglieder folgt gem. Art. O EUV, ähnlich wie auch die Vertragsänderung nach Art. N EUV, in einem durch den Unionsvertrag festgelegten Verfahren und setzt die

48

Siehe hierzu E. Klein (oben Fn. 28), Art. O, Rn. 12; C.D. Ehlermann, Mitgliedschaft in der Europäischen Gemeinschaft - Rechtsprobleme der Erweiterung, der Mitgliedschaft und der Verkleinerung, EuR 1984, S. 113 ff, 116; P. Dagtoglou, Die Süderweiterung der Europäischen Gemeinschft -insbesondere der Beitritt Griechenlands, EuR 1980, S. 1 ff, 8. 
Mitwirkung von Gemeinschaftsorganen voraus. Danach sollte es möglich sein, die nach Art. O Abs. 1 EUV für die Aufnahme eines neuen Mitgliedes erforderlichen Beschlüsse des Rates und die Zustimmung des Europäischen Parlaments als "Handlungen" dieser Organe gem. Art. 173 Abs. 1 EGV anzufechten. Der Begriff Handlungen im Sinne dieses Artikels ist nach der Rechtsprechung des Gerichtshofes nicht auf die Maßnahmen gem. Art. 189 EGV beschränkt. Er ist vielmehr weit auszulegen und umfaßt alle Handlungen, die dazu bestimmt sind, Rechtswirkungen zu erzeugen, ohne Unterscheidung ihrer Rechtsnatur oder Form. ${ }^{49}$ Für eine gerichtliche Überprüfung der vom Rat und dem Parlament gemäß Art. O EUV zu treffenden Beschlüsse spricht auch, daß Art. L lit. c EUV die Rechtsprechungsgewalt des Gerichtshofes ausdrücklich auch auf Art. L bis S und damit auch Art. O EUV ausdehnt. Diese Norm wäre sinnlos, wenn sich nicht auch eine einschlägige Verfahrensart finden ließe.

Möglich wäre auch die Überprüfung der Gültigkeit der Ratsbeschlüsse und der Zustimmung des Parlamentes im Rahmen eines Vorabentscheidungsverfahrens nach Art. 177 lit. b EGV. Auch hier ist der Begriff "Handlungen der Organe" weit auszulegen. ${ }^{50}$ Das Urteil in der Rechtssache Mattheus/Doego ${ }^{51}$ steht dem nicht unbedingt entgegen. In diesem Vorabentscheidungsverfahren war der Gerichtshof aufgefordert worden, noch vor Abschluß der Beitrittsakte mit Griechenland, Portugal und Spanien zu der Frage Stellung zu nehmen, ob das Gemeinschaftsrecht materiellrechtliche Grenzen für den Beitritt von Drittstaaten zu den Europäischen Gemeinschaften enthält und welche das seien. Der Gerichtshof hat es jedoch abgelehnt, sich hierzu in der Sache zu äußern und sich mit folgender Begründung für unzuständig erklärt: "[Art. 237 legt] für die Aufnahme neuer Mitgliedstaaten ein genaues und wohlabgegrenztes Verfahren fest, in welchem die Beitrittsbedingungen von den in dem Artikel selbst genannten Stellen zu erarbeiten sind, [...] ohne daß es möglich wäre, ihren Inhalt vorweg unter Einschaltung der Gerichte festzulegen. Der Gerichtshof kann sich deshalb nicht im Rahmen eines Verfahrens nach Art. 177 EWG-Vertrag zu Form und Inhalt der gegebenenfalls festzulegenden Bedingungen äußern." ${ }^{52}$ Damit hat sich der Gerichtshof immerhin die Möglichkeit offengelassen, nach Abschlu $\beta$ der Beitrittsverhandlungen zur Rechtmäßigkeit der Beitrittsverträge Stellung zu nehmen.

Vgl. F. H. Wenig, in: Grabitz/Hilf (oben Fn. 33), Art. 173, Rn. 7; EuGH Rs. 22/70 (AETR), Slg. 1971, S. 263 ff., 277

Vgl. J. Wohlfahrt, in: Grabitz/Hilf (oben Fn. 33), Art. 177 Rn. 17; Rs 9/73 (Schlüter), Slg. 1973, S. $1135 \mathrm{ff}$.

51 Rs 93/78 (Mattheus/Doego), Slg. 1978, S. 2203 ff.

52 A.a.O.S. 2211 , Rn. 8;

53

Siehe auch $C$. D. Ehlermann, Mitgliedschaft in der Europäischen Gemeinschaft -Rechtsprobleme der Erweiterung, der Mitgliedschaft und der Verkleinerung, EuR 1984, S.113 ff, 116. 
Fraglich bleibt aber, ob mit Art. L EUV nur die Einhaltung der wesentlichen Verfahrensvorschriften des Artikel O EUV (Anhörung der Kommission, Zustimmung des Europäischen Parlaments, Beschluß des Ministerrates) gerichtlich überprüft werden sollen, oder ob der Gerichtshof auch befugt sein soll, die Einhaltung materieller Voraussetzungen der Aufnahme neuer Mitglieder zu überprüfen. Die Anknüpfung beispielsweise an den Rechtsakt des Ratsbeschlusses erscheint zwar formal möglich, darf aber nicht darüber hinwegtäuschen, daß es in der Sache letztlich doch um die gerichtliche Überprüfung von Primärrecht aus den Beitrittsverträgen gehen soll. Hier besteht ein enger Zusammenhang mit der Frage, ob das Gemeinschaftsrecht überhaupt Maßstäbe für eine solche Überprüfung bereithält, ob es, mit anderen Worten, so etwas wie "materielle Grenzen der Vertragsänderung" gibt. Hierzu müßte der Gerichtshof seine im EWR-Gutachten ${ }^{54}$ entwickelte Rechtsprechung zu einem "änderungsfesten Kern" des EGV, der die Grundlagen der Gemeinschaft selbst absichern soll, fortsetzen. ${ }^{55}$ Das dürfte mit Blick auf die oben genannten Voraussetzungen der Erweiterung möglich sein, da sie direkt den Legitimationsgrundlagen der Gemeinschaft und deren Funktionsbedingungen entnommen werden können. Für die Annahme von Normenhierarchien innerhalb des Primärrechts spricht die in Maastricht eingefügte Revisionsklausel des Art. N EUV, dessen Absatz 2 die Mitgliedstaaten bei der Prüfung einer Vertragsrevision auf die in Art. A und B EUV niedergelegten Unionsziele verpflichtet. ${ }^{56}$ Für Beitrittsverträge, die in ihrer materiell vertragsändernden Wirkung unterhalb der Schwelle der Vertragsrevision bleiben, sollte diese Verpflichtung erst recht gelten.

Eine andere Frage ist die der Kontrolldichte. Hinsichtlich aller Voraussetzungen ist ein mehr oder weniger großer Beurteilungsspielraum der politischen Organe zu bejahen. Das ergibt sich aus der Sachnatur der Kriterien wie dem politischen Charakter des Erweiterungsprozesses. ${ }^{57}$ Wie aus den zu den jeweiligen Kriterien gemachten Ausführungen bereits ersichtlich, kann die Kontrolldichte durchaus variieren. Am weitesten dürfte die Überprüfungskompetenz des Gerichtshofs bei den verfassungsstaatlichen Anforderungen der Ziffer 1 der Kopenhagener Kriterien sein, am geringsten bei den Entscheidungen, denen ökonomische und politische Prognosen zugrunde liegen. Hier mag die Überprüfungskompetenz de facto auf Null reduziert sein.

54

55

56

57
Gutachten 1/91, Slg. 1991 I-, S. 6079 ff.

Siehe hierzu E. Klein, in: Hailbronner u.a. (Hrsg.), Handkommentar zum EUV/EGV, 3. Lieferung August 1994, Art. N, Rn. 15; U. Everling, Zur Stellung der Mitgliedstaaten der Europäischen Union als "Herren der Verträge", in: FS für Rudolf Bernhardt, 1995, S. $1161 \mathrm{ff}$.

Siehe zur Frage von Normenhierarchien innerhalb des Primärrechts $R$. Bieber, Les limites matérielles et formelles à la révision des traités établissant la communauté européenne, RMC 1993, S. 343 ff. ders. / I. Salomé, Hierarchy of Norms in European Law, CMLR 1996, S. 907 ff. (bejahend). M. Heintzen, Hierarchisierungsprozesse innerhalb des Primärrechts der Europäischen Gemeinschaft, EuR 1994, S. 35 ff. (verneinend).

Vedder (oben Fn. 33), Art. 237 Rn. 5; Ehlermann, a.a.O.; E. Klein (oben Fn. 28), Art. O Rn. 12 äußern sich daher skeptisch bezüglich der Justiziabilität der Beitrittskriterien. 


\section{Rechtsf ragen der aktuellen Erweiterungsdebatte - "Osterweiterung"}

\section{Beitrittsfähigkeit der mittel- und osteuropäischen Staaten}

\section{a) Institutionelle Stabilität. Rechtsstaatlichkeit, Demokratie}

Eine Feststellung der Beitrittsfähigkeit der Antragsteller muß sich an der tatsächlichen Lage orientieren; formale Bekenntnisse und Verfassungsproklamationen genügen nicht. Bei der in der Agenda 2000 vorgenommenen Beurteilung ${ }^{58}$ ist die Kommission zu dem Schluß gekommen, daß, auch wenn in mehreren beitrittswilligen Ländern noch Fortschritte bei der effektiven Ausübung der Demokratie und beim Schutz der Minderheiten gemacht werden müssen, nur ein Staat die vom Europäischen Rat in Kopenhagen festgesetzten politischen Kriterien nicht erfüllt. ${ }^{59}$ Angesichts dieses Befundes dürfte die Mitgliedschaft der Kandidaten insoweit keine allzu großen Probleme aufwerfen. Für den Fall nachlassender Bemühungen der Neumitglieder (aber auch der alten) hat der Amsterdamer Vertrag Vorsorge getroffen. Mit einem neuen Artikel 7 (ex-Art. F.1) EUV werden Sanktionsmöglichkeiten gegenüber solchen Mitgliedstaaten geschaffen, die die gemeinschaftsrechtlichen Standards der Demokratie, der Rechtsstaatlichkeit und der Achtung der Menschenrechte mißachten. Nach dieser Bestimmung kann der Rat, der in der Zusammensetzung der Staats- und Regierungschefs tagt, durch einstimmigen Beschluß und nach Zustimmung des Europäischen Parlaments feststellen, daß ein Mitgliedstaat die in Art. 6 (ex-Art. F) Abs. 1 EUV genannten Grundsätze anhaltend und in schwerwiegender Weise verletzt. ${ }^{60}$ In einem weiteren Schritt kann der Rat mit qualifizierter Mehrheit, ohne Berücksichtigung der Stimmen des betreffenden Mitgliedstaates, ${ }^{61}$ die Aussetzung bestimmter vertraglicher Rechte beschließen. ${ }^{62}$ Eine derartige "verfassungsstaatliche Konditionalisierung" von Mitgliedschaftsrechten ist bislang ausdrücklich erst bei der Revision des Lomé-Abkommens 1995 (Lomé V) erreicht worden. ${ }^{63}$ Allerdings hat die Gemeinschaft auch ohne eindeutige Rechtsgrundlage

Bei den Stellungnahmen der Kommission zur Beitrittsfähigkeit der Kandidaten in der Agenda 2000 handelt es sich nicht um die nach Art. O Abs. 1 erforderliche Anhörung der Kommission. Seit den 1970 begonnenen Verhandlungen über den Beitritt Großbritanniens, Irlands und Dänemarks hat sich über den Wortlaut des damals maßgeblichen Art. 237 EWGV ein mehrstufiges Verfahren entwickelt (vgl. Vedder (oben Fn. 33), Rn. 10 ff.) Danach gehen den eigentlichen Beitrittsverhandlungen eine vorläufige Stellungnahme der Kommission sowie ein Ratsbeschluß über die Aufnahme von Verhandlungen voraus. In dieser Einleitungsphase befindet sich der Proze $\beta$ der Osterweiterung derzeit.

60

61

62

62

63

Slowakei. Siehe Agenda 2000 (oben Fn. 1), S. 45.

Art. 7 (ex-Art. F.1) Abs. 1 EUV.

Art. 7 (ex-Art. F.1) Abs. 4 EUV.

Art. 7 (ex-Art. F.1) Abs. 2 EUV.

Vgl. G. Nicolaysen, Europarecht II - Das Wirtschaftsrecht im Binnemarkt, 1996, S. 523 m.w.Nachw. 
während der griechischen Militärdiktatur 1967-74 Finanzhilfen aus dem Assoziationsabkommen "bis zur Rückkehr zu einem normalen demokratischen Leben" ausgesetzt. ${ }^{64} \mathrm{Ob}$ durch Artikel 7 (ex-Art. F.1) EUV ähnliche finanzielle Sanktionen auch gegenüber Mitgliedstaaten ermöglicht werden, erscheint indes noch zweif elhaft. Soweit Leistungen etwa aus den Strukturfonds unmittelbar einzelne Personen oder Unternehmen begünstigen, stellt sich die bislang noch ungeklärte Frage, ob diese sich nicht gegen eine Streichung der Fördermittel auf die ebenfalls gemeinschaftsrechtlich verbürgten Grundsätze der Rechtssicherheit und des Vertrauensschutzes berufen können. Es wäre paradox, im Namen der Durchsetzung von Rechtsstaatlichkeit, Demokratie und Menschenrechten gerade denjenigen zu schaden, denen diese Grundsätze dienen sollen. Daher sieht der in Amsterdam neu geschaffene Art. 309 (ex-Art. 236) Abs. 2 EGV in einer salvatorischen Klausel vor, daß der Rat bei der Aussetzung von Mitgliedschaftsrechten "die möglichen Auswirkungen einer solchen Aussetzung auf die Rechte und Pflichten natürlicher und juristischer Personen" berücksichtigt.

\section{b) Binnenmarktreife und Integrationswilligkeit}

Wie oben dargelegt, setzt eine Mitgliedschaft rechtlich nicht eine vollständige Wettbewerbsfähigkeit voraus, wie die Kopenhagener Kriterien dies nahezulegen scheinen. Schon bei früheren Beitritten wurde der Schwergewicht auf einen möglichst raschen Beitritt gelegt und den anfälligen Sektoren der Wirtschaft mit Übergangsfristen geholfen. $\mathrm{Zu} \mathrm{Recht} \mathrm{hat}$ die Kommission daher bei ihrer Evaluierung lediglich geprüft, ob die Volkswirtschaften der beitrittswilligen Länder ein Minimum an Wettbewerbsfähigkeit erreicht haben und die Möglichkeit künftiger Entwicklungen mit einbezogen. ${ }^{65}$ Aber auch hier sind Zweifel angebracht. ${ }^{66}$ Ungeachtet der anzuerkennenden Anstrengungen, auch der Hilfe, die insoweit seitens der Gemeinschaft geboten wird ("Pre-accession"-Strategie), sind die Rückstände doch noch erheblich. In ihrer volkswirtschaftlichen Leistungsfähigkeit liegen die mittelund osteuropäischen Staaten nach wie vor weit hinter dem EU-Durchschnitt zurück. Das durchschnittliche Pro-Kopf-BIP der Kandidaten liegt noch immer bei etwa einem Drittel des EU-Durchschnitts. Wirtschaftlich betrachtet, ist gegenwärtig keines der zehn Reformländer wirklich "binnenmarktfähig". 67 Auch die Entwicklung der für die Um- und Durch-

64 65

66

67

Vgl. hierzu T. Oppermann, Europarecht, 1991, Rn. 1872.

Vgl. Agenda 2000, S. $46 \mathrm{ff}$.

Siehe in der wirtschaftswissenschaftlichen Literatur etwa S. Franke, Europa vom Atlantik bis zum Ural - Vision, Illusion oder absehbare Realität?, Wirtschaftsdienst 1997, Heft 10, S. 573-579.

Vgl. Agenda 2000, S. 47. Die dort vorgenommenen wirtschaftlichen Einschätzungen der Kommission sind allerdings bereits Gegenstand politischer Auseinandersetzungen geworden. Lettland und Litauen beispielsweise tragen vor, daß teils fehlerhafte, teils veraltete Daten verwendet worden seien (vgl. F.A.Z. v. 10. Sept. 1997, S. 10 und 14. Okt. 1997, S. 7). 
setzung des Gemeinschaftsrechts notwendigen rechtsstaatlichen Verfahren und Strukturen läßt noch zu wünschen übrig.

Eine rasche Aufnahme der am meisten entwickelten fünf Länder wird daher wohl nur unter erheblichen Abstrichen gegenüber den normalen Rechten und Pflichten einer EU-Mitgliedschaft möglich sein. Dabei sind beide Seiten betroffen: Während die wirtschaftliche Situation der meisten mittel- und osteuropäischen Staaten an lange Übergangsfristen für den Warenverkehr mit vielen industriellen Erzeugnissen denken läßt, verlangen auf Seiten der Gemeinschaft die Bereiche Stahl und Textil nach Schutz. Fraglich ist weiterhin, ob die ebenfalls in der Agenda 2000 vorgesehenen Reformen der Gemeinsamen Agrarpolitik weitreichend genug sind und schnell genug greifen, so daß sich die Union eine Erweiterung um große landwirtschaftliche Produzenten leisten kann. Schon regt sich hierzulande heftiger Widerstand der Agrarlobby gegen die Vorstellungen der Kommission. Hinzu kommen die angesichts der tatsächlichen Migrationsstatistiken innerhalb der Union nicht immer ganz nachvollziehbaren Sorgen der Mitgliedstaaten vor einer Überschwemmung mit Wanderarbeitnehmern. Der sensible Bereich des Personenverkehrs lädt zu innovativen Sonderregelungen ein. Diskutiert werden umfangreiche Übergangsregelungen, eventuell mehrfach verlängerbar oder gar zeitlich unbefristet, die in ihrer Wirkung Bereichsausnahmen nahe kommen. Die Rede war auch schon von "Teilbeitritten", durch die politisch und/oder wirtschaftlich heikle Integrationsbereiche völlig ausgeklammert werden könnten.

\section{(1) Teilbeitritte}

Teilbeitritte, wie sie in der Vergangenheit etwa von dem damaligen, für die Außenbeziehungen der Gemeinschaft zuständigen Vize-Präsidenten der Kommission, Frans Andriessen, in die Diskussion eingebracht worden sind, ${ }^{68}$ sind mit dem geltenden Gemeinschaftsrecht eindeutig nicht zu vereinbaren. Gegen eine solche Lösung spricht schon Art. O EUV selbst, der, wie bereits erwähnt, nur noch einen Beitritt zur Union als Ganzer vorsieht. Ein wie immer gearteter Teilbeitritt wäre aber auch mit anderen Normen des Gemeinschaftsrechts nur schwer zu vereinbaren. Nach Artikel C Abs. 1 EUV verfügt die Union über "einen einheitlichen institutionellen Rahmen, der die Kohärenz und Kontinuität der Maßnahmen zur Erreichung ihrer Ziele unter gleichzeitiger Wahrung und Weiterentwicklung des gemeinschaftlichen Besitzstandes sicherstellt". Dieser institutionelle Rahmen würde aber gesprengt, wollte man neue Mitglieder nur für bestimmte Bereiche der Unionspolitiken zulassen. Auch wäre der Grundsatz von der vollen Wahrung und Weiterentwicklung

68

F. Andriessen, Towards a Community of Twenty-Four?, Speech held at the 69th Plenary Assembly of the Eurochambers, Economic ans Social Committee, Brussels, 19 April 1991 (Mimeo.): "Af filiate membership would provide membership rights and obligations in some areas, while excluding others, at least for a tranisitional period." Ähnlich T. Pedersen, The Common Foreign and Security Policy and the Challenge of Enlargement, in: Norgaard/Pedersen/Petersen (Hrsg.), The European Community in World Politics, 1993, S. 38. 
des gemeinschaftlichen Besitzstandes, der sich als Ziel der Union auch in Art. B, 5. Spiegelstrich EUV wiederfindet, in Frage gestellt, da ein Wesensmerkmal des acquis gerade seine gleiche Geltung in allen Mitgliedstaaten ist. Schließlich wäre auch die in Art. A Abs. 3 EUV niedergelegte Aufgabe der Union, "die Beziehungen zwischen den Mitgliedstaaten sowie zwischen ihren Völkern kohärent und solidarisch zu gestalten", wohl kaum noch zu erfüllen.

\section{(2) Übergangsfristen}

Von größerer praktischer Bedeutung ist die Frage der Übergangsfristen. Auch hier gilt der im Maastrichter Vertrag mehrfach verankerte Grundsatz der Sicherstellung der Wahrung und Weiterentwicklung des gemeinschaftlichen Besitzstandes. Der bereits erwähnte Artikel C Abs. 1 EUV, der sich seinem Wortlaut nach zunächst nur auf die institutionelle Ausgestaltung der Union zu beziehen scheint, ist auch als Bestandsgarantie für den bereits erreichten (materiellen) Integrationsstand aufzufassen. ${ }^{69}$ Denn eine der Aufgaben des durch diese Bestimmung geforderten einheitlichen institutionellen Rahmens ist gerade die "gleichzeitige Wahrung und Weiterentwicklung des gemeinschaftlichen Besitzstandes". Deutlicher noch schützt Art. B EUV den acquis communautaire, nach dessen 5. Spiegelstrich es eines der Ziele der Union ist, "die volle Wahrung des gemeinschaftlichen Besitzstandes und seine Weiterentwicklung [...] sicherzustellen". Als Bestands- und Entwicklungsgarantie erschöpft sich der Grundsatz von der Wahrung des Besitzstandes nicht in einer bloßen Zielvorgabe, sondern bindet die Gemeinschaft in ihren gesamten Aktivitäten, damit auch in der Erweiterungspolitik. Die Grenze für die mögliche Rechtsentwicklung auf der Grundlage des Unionsvertrages ist dort erreicht, wo der bereits erreichte Entwicklungsstand aufgegeben würde. ${ }^{70}$ Eben dies wäre aber bei unangemessen langen, mehrfach verlängerbaren oder gar unbefristeten Übergangsregelungen der Fall, da sie de facto die Wirkungen von Teilbeitritten hätten. Abweichungen neu beitretender Staaten vom Gemeinschaftsrecht sind daher nur in Form zeitlich begrenzter Übergangsfristen zulässig. ${ }^{71}$ Erforderlich werdende Sonderregelungen zugunsten (oder zu Lasten) der neu beitretenden Transformationsländer lassen sich nur mit den tradierten Instrumenten zeitlich befristeter Übergangsregelungen bewerkstelligen.

Eine weitere Frage ist, in welchen Bereichen Übergangsfristen überhaupt zulässig sind. Die durch den Amsterdamer Vertrag vorgenommene Verankerung verfassungsstaatlicher Grundanforderungen in der Beitrittsklausel des neuen Art. 49 (ex-Art. O) EUV zeigt, daß jedenfalls in diesem Bereich strenge Maßstäbe anzulegen sind. Spätestens zum Zeitpunkt 
des Beitritts können vom Erfordernis der demokratischen Ordnung, der Rechtsstaatlichkeit und der Achtung der Menschen- und Minderheitsrechte keine Abstriche gemacht werden. Ähnliches dürfte für diejenigen Anforderungen an die Binnenmarktreife der Fall sein, die sich auf die Wirtschaftsverfassung beziehen, also für die Frage, ob mit der Rechts- und Verwaltungsordnung die innerstaatlichen Voraussetzungen für die Übernahme und vor allem die Anwendung des Gemeinschaftsrechts vorliegen. Zulässige Übergangsfristen werden sich daher auf den Bereich der wirtschaftlichen Verträglichkeit einer Übernahme des Gemeinschaftsrechts beschränken, etwa im Warenverkehr. In dem durch die Übernahme des Schengen-Acquis in den ersten Pfeiler besonders sensibel gewordenen Gebiet des freien Personenverkehrs sind bereits beim Beitritt Spaniens und Portugals schon Übergangsfristen von 10 Jahren vereinbart worden. Da auch die gegenwärtigen Mitgliedstaaten, beispielsweise Griechenland, noch nicht alle voll am Schengener System teilnehmen bzw. Ausnahmen und Sonderregeln für sich in Anspruch nehmen, wie das Vereinigte Königreich, Irland und Dänemark, dürften Übergangslösungen auch hier möglich sein.

\section{(3) Flexibilitätsklausel}

Die unter diesem Stichwort zusammengefaßten neuen Regelungen in EUV und EGV ${ }^{72}$ gestatten im Geltungsbereich des EGV und des sog. 3. Pfeilers, also der Zusammenarbeit in den Bereichen Justiz und Inneres, eine "engere Zusammenarbeit" zwischen den einzelnen Mitgliedstaaten. Sie legitimieren also eine "vorauseilende Integration" einer Gruppe von Mitgliedstaaten, wenn andere an diesem Schritt noch nicht teilnehmen wollen oder können. Diese "Flexibilität" der Integration, die dem Grunde nach schon im Maastrichter Vertrag präjudiziert worden ist (Sozialprotokoll, Wirtschafts- und Währungsunion), unterliegt aber wichtigen Einschränkungen: Die engere Zusammenarbeit muß von einer Mehrheit der Mitgliedstaaten getragen sein; sie darf den Besitzstand der Gemeinschaft nicht beeinträchtigen und muß allen Mitgliedstaaten offenstehen. ${ }^{73}$ Im Bereich des EGV ist die "engere Zusammenarbeit" zudem an das alleinige Vorschlagsrecht der Kommission gebunden; ${ }^{74}$ außerdem kann diese über einen späteren Beitritt weiterer Mitgliedstaaten zur Zusammen-

Art. 40 (ex-Art. K.12) ff., Art. 43 (ex-Art. K.15) ff. EUV; Art. 11 (ex-Art. 5 a) EGV. Siehe zu diesen Änderungen C. D. Ehlermann, Rechtliche Grenzen eines Europas in mehreren Geschwindigkeiten und unterschiedlichen Gruppierungen, EuR 1997, Heft 4; W. Wessels, Der Amsterdamer Vertrag - Durch Stückwerksreformen zu einer effizienten, erweitertern und föderalen Union?, integration 1997, S. 117 ff, 128 f.; P. Huber, Differenzierte Integration und Flexibiblität als neues Ordnungsmuster der Europäischen Union?, EuR 1996, S. 347 ff.; C. Giering, Vertiefung durch Differenzierung - Flexibilisierungskonzepte in der aktuellen Reformdebatte, integration 1997, S. 72 ff.; Grundlegend E. Grabitz (Hrsg.), Abgestufte Integration - Eine Alternative zum herkömmlichen Integrationskonzept?, 1984. 
arbeit ohne den Rat entscheiden. ${ }^{75}$ Damit ist klargestellt: Die durch den Amsterdamer Vertrag neu eingeführten Bestimmungen zur engeren Zusammenarbeit werden kein Instrument zur Legitimierung von unbegrenzten Übergangsfristen, Bereichsausnahmen, wie immer gearteten Teilbeitritten oder gar "Mitgliedschaften zweiter Klasse" unterhalb der Schwelle des beim Beitritt erreichten acquis sein. Eine rückwärtsgerichtete "flexibilité à rebours" ist und bleibt mit dem Gemeinschaftsrecht unvereinbar. Entsprechende Arrangements könnten nur unterhalb der Schwelle der Mitgliedschaft getroffen werden. Hierzu stehen ausreichend Instrumente zur Verfügung, z.B. Assoziationsabkommen.

Vor allem dürfte aus dem Erfordernis der Offenheit der "engeren Zusammenarbeit" für alle Mitgliedstaaten zu schließen sein, daß die Flexibilitätsklausel keinerlei Abstriche vom Erfordernis der Beitrittsfähigkeit und Integrationswilligkeit der Kandidaten zuläßt. Es darf, mit anderen Worten, auch nach Inkrafttreten der Bestimmung kein Neumitglied aufgenommen werden, von dem schon heute feststeht, daß es an gewissen, vielleicht noch nicht abschließend definierten, aber im Kern doch schon angelegten Zielen der Union niemals wird teilnehmen können.

\section{Erweiterungsfähigkeit der Union}

Eines der wesentlichen Ziele des Gipfels von Amsterdam war die Vorbereitung der Union auf die bevorstehenden Erweiterungsrunden. ${ }^{76}$ Dieses Ziel wurde nur teilweise erreicht. ${ }^{77}$ Die wenigen greifbaren Ergebnisse des Gipfels zu den Institutionen sind in einem "Protokoll über die Organe im Hinblick auf die Erweiterung der EU" zusammengefaßt, das dem Vertrag von Amsterdam beigefügt ist. ${ }^{78}$ Nach dessen Artikel 1 wird die Kommission mit Inkrafttreten der ersten Erweiterung aus nur noch jeweils einem Kommissar pro Mitgliedstaat bestehen. Die Regelung setzt allerdings voraus, daß die Frage der Stimmengewichtung im Rat bis zu diesem Zeitpunkt in einer "für alle Mitgliedstaten annehmbaren Weise" gelöst ist. Als Möglichkeiten werden sowohl eine Neugewichtung der Stimmenverhältnisse als auch die Einführung einer doppelten Mehrheit genannt. Besonders erwähnt wird auch die Frage einer Kompensation für diejenigen Mitgliedstaaten, die bisher zwei Kommissare stellen durften, also Deutschland, Italien, Frankreich, Spanien und Großbritannien. Daß hiermit noch kein substantieller Fortschritt bei der Frage einer institutionellen Beitrittsfähigkeit erzielt worden ist, war auch den Staats- und Regierungschefs bewußt.

75

76

77

78

Art. 11 (ex-Art. 5 a) Abs. 3 EGV.

Vgl. zu den verschiedenen Vorschlägen für institutionelle Reformen u.a. U. Fastenrath, Die Struktur der erweiterten Europäischen Union, EuR Beiheft 1, 1994, S. $101 \mathrm{ff}$

Siehe zu einer ersten politikwissenschaftlichen Bewertung des Amsterdamer Gipfels W. Wessels (oben Fn. 72).

Siehe Vertrag von Amsterdam (oben Fn. 9), S. 138 f. 
Artikel 2 des Protokolls sieht daher gleich ein "Amsterdam II" vor: Spätestens ein Jahr bevor die Zahl der Mitgliedstaaten die Zwanzig überschreiten wird, soll eine neue Regierungskonferenz einberufen werden, um "Zusammensetzung und Arbeitsweise der Organe umfassend zu überprüfen".

Damit hat der Amsterdamer Gipfel zwei der politisch umstrittensten Fragen, nämlich die der zukünftigen Stimmengewichtung im Rat und der Zusammensetzung der Kommission, d.h., die Balance zwischen großen und kleinen, neuen und alten Mitgliedstaaten, auf die kommenden Beitrittsverhandlungen verschoben. Es besteht die Gefahr, daß die ohnehin aufwendigen und komplizierten Verhandlungen von Querelen über das Abstimmungsverfahren überschattet werden. Selbst ein Scheitern der Beitritte an dieser Frage, das wegen eines britischen Vetos schon in der letzten Erweiterungsrunde kurzzeitig gedroht hatte, ist nicht völlig auszuschließen. Sollten die Beitrittsverhandlungen mit den von der Kommission benannten fünf mittel- und osteuropäischen Kandidaten und Zypern zugleich abgeschlossen werden können, was angesichts des andauernden Konflikts zwischen den griechischen und türkischen Bevölkerungsteilen der Insel allerdings wenig wahrscheinlich ist, so würde sich immerhin die Chance ergeben, auf der neuen Regierungskonferenz (Amsterdam II) zu einer umfassenden Neuordnung des institutionellen Gefüges zu gelangen. Die Vereinbarung, daß diese Neuverhandlung erst bei Überschreiten der Mitgliederzahl von zwanzig stattfinden soll, zeigt zugleich, daß die Staats- und Regierungschefs nicht von einer baldigen Lösung des Zypern-Problems ausgegangen sind.

Offenbar war man in Amsterdam aber der Ansicht, daß die gegenwärtigen Strukturen, die einst für die Gemeinschaft der Sechs geschaffen wurden, immerhin noch mit zwanzig Mitgliedern funktionieren werden. Dies könnte sich als folgenschwerer Fehler erweisen. Ob die Gemeinschaft die in Aussicht genommene Osterweiterung ohne erhebliche Nachbesserungen des mageren Amsterdamer Ergebnisses wirklich verkraften kann, scheint nach wie vor eine offene Frage zu sein. Verschiedene schwierige Fragen wie etwa das Sprachenregime, ${ }^{79}$ sind auf der Konferenz überhaupt nicht erörtert worden. Zwar konnten in anderen institutionellen Bereichen gewisse Fortschritte erzielt werden, beispielsweise wurden die Rechte des Europäischen Parlaments durch eine Ausdehnung des Mitentscheidungsverfahrens verbessert und die Stellung des Kommissionspräsidenten dadurch gestärkt, daß dieser in Zukunft bei der Auswahl der Kommissare mitentscheiden darf und eine Art Richtlinienkompetenz nach innen erhält. ${ }^{80}$ - Die Frage, ob die Union wirklich reif für die Aufnahme neuer Mitglieder ist, muß aber nach wie vor als offen angesehen werden.

Dazu demnächst T. Bruha, Rechtliche Aspekte der Vielsprachigkeit: Vertrags-, Amts-, Arbeitsund Verkehrssprachen in der Europäischen Union, in: ders./H.-J. Seeler (Hrsg.), Die Europäische Union und ihre Sprachen, Baden-Baden, 1998.

80

Siehe Art. 214 (ex-Art. 158) Abs. 2 und 219 (ex-Art. 163) EGV. 


\section{Ansprüche auf Mitgliedschaft}

Ist eine Erweiterung der Union somit an die doppelte Voraussetzung der Beitrittsfähigkeit (der Kandidaten) und der Erweiterungsfähigkeit (der Union) gebunden, so stellt sich die ganz andere Frage, ob die Gemeinschaft bei Vorliegen dieser Voraussetzungen politisch frei über den Antrag entscheiden kann, oder ob sie hierbei rechtlichen Bindungen unterliegt. Diese Frage ist zur Zeit zugegebenermaßen mehr theoretischer Natur, denn daß sie wirklich praktische Bedeutung hätte. In den tatsächlich oder potentiell in Betracht kommenden Fällen (etwa die oben als "Grenzfälle bezeichneten Beitritte Rußlands und der Türkei oder auch europäischer "Mikrostaaten") steht immer auch die Systemverträglichkeit derartiger Beitritte in Frage. So ist der institutionelle Rahmen der EU, insbesondere was die Mitwirkung in Rat und Kommission mit ihren zahlreichen Untergliederungen betrifft, nicht auf Kleinststaaten zugeschnitten. Die Aufnahme Rußlands oder der Türkei dürfte sich in besonderer Weise auf die politische Finalität des Einigungsprozesses auswirken. Die Erweiterungsvoraussetzungen liegen in solchen Fällen eben nicht ohne weiteres vor. Ansprüche auf Mitgliedschaft in der Europäischen Union, wie sie in der Vergangenheit etwa für gewisse internationale Organisationen diskutiert, aber überwiegend abgelehnt worden sind, scheiden daher aus. Dies gilt um so mehr, als die Gemeinschaft in ihren jetzigen Strukturen die Grenze des Wachstums erreicht hat. Neubeitritte erfordern Reformen, über die politisch zu entscheiden ist. Zu fragen wäre auch, ob die Gemeinschaft bezüglich einer etwaigen Problematik von Mitgliedschaftsansprüchen Dritter nicht bereits mehr mit einem Staat, dem gegenüber es keine Beitrittsansprüche gibt, als mit einer internationalen Organisation zu vergleichen wäre. Das mag aber dahinstehen. Selbst wenn man die Gemeinschaft in der völkerrechtlichen Terminologie als "beschränkt offene" oder gar "notwendige regionale Organisation" ${ }^{18}$ bezeichnen und qualifizieren würde, ergäben sich daraus allenfalls Pflichten zur wohlwollenden Prüfung eingegangener Beitrittsanträge. Damit wäre im praktischen Ergebnis aber kaum etwas gewonnen.

Andererseits ist die Gemeinschaft aber, wie bereits ausgeführt, auf einen "immer engeren Zusammenschluß der europäischen Völker" (Präambel des EGV, Abs. 1) hin angelegt. Die Union ist ausdrücklich gehalten, nach der historischen Überwindung der Teilung des europäischen Kontinents "feste Grundlagen für die Gestalt des zukünftigen Europa zu schaffen" (Präambel EUV, Abs. 2). Daraus wird man zwar keine Ansprüche auf Mitgliedschaft in der Union ableiten können, wohl aber "Partizipationsansprüche" am europäischen Integrationsprozeß. Dieser Prozeß ist in einem gesamteuropäischen Sinne zu verstehen und sollte weit gefaßt werden, sowohl was den Kreis der in Betracht kommenden Mitgliedstaaten als auch was den Grad der "Offenheit" der Union betrifft: Alle europäischen Staaten, ob klein ob groß, ob West ob Ost, ob (mehr) christlich oder (mehr) islamisch, haben legitime Anwart-

Siehe zu dieser These T. Oppermann, Rechtsfragen der Mitgliedschaft in internationalen Organisationen, in: Berichte der Deutschen Gesellschaft für Völkerrecht, Heft 17 (1975), S. 53 ff., 66-75. 
schaften auf Einbeziehung in den "Bau Europas". Wo die EU-Mitgliedschaft unter den gegebenen Umständen das adäquate und auch mit der Fortentwicklung der Gemeinschaft verträgliche Partizipationsniveau ist, ist diese in Betracht zu ziehen. Wo ein Beitritt zur Gemeinschaft aus den einen oder anderen Gründen ausscheidet, ist eine Partizipation auf der nächstniedrigeren Integrationsstufe in Aussicht zu stellen (Europäischer Wirtschaftsraum, Zollunion, Europaabkommen, Freihandelsabkommen, Partnerschafts- und Kooperationsabkommen) ${ }^{82}$. Stets sollte die Gemeinschaft gehalten sein, einer "Höherstufung" positiv gegenüber zu stehen, wenn dies gewünscht wird, möglich ist und mit den Zielen der Gemeinschaft in Übereinstimmung gebracht werden kann. Auf welcher Stufe "Partizipationsansprüche" auch immer geltend gemacht werden: Diesbezügliche Entscheidungen der Gemeinschaft unterfallen nicht dem Bereich des freien politischen Ermessens, ${ }^{83}$ sie sind rechtlich "zielgebunden" wenn auch naturgemäß nicht justiziabel.

\section{Erweiterung und Vertief ung}

Auch bei der Vermittlung zwischen den gleichermaßen vorgegebenen Zielen der Erweiterung und Vertiefung der Europäischen Union und des Integrationsprozesses agieren Mitgliedstaaten und Gemeinschaftsorgane nicht in einem rechtlichen Freiraum. So dürfte sich die Gemeinschaft im Interesse einer möglichst "großen" Union eben nicht in eine paneuropäische Freihandelszone verwandeln. Dem stehen die materiellen Integrationsziele und der Grundsatz der Wahrung des acquis communautaire entgegen. Doch liegt hier auch nicht das eigentliche Problem. Die in politisch-rhetorischer Überspitzung an die Wand gemalte Gefahr einer Denaturierung der Gemeinschaft zu einer bloßen Freihandelszone besteht realiter nicht. Niemand will einen solchen Rückschritt, auch nicht die Briten.

Die eigentliche Brisanz des Zielkonfliktes zwischen Erweiterung und Vertiefung eröffnet sich statt dessen in der Perspektive der Fortentwicklung der Gemeinschaft. Hier ein immanentes Spannungsverhältnis zu leugnen, wäre unredlich. Selbstverständlich wirken sich Zahl, geographische Lage, ethnische Zusammensetzung, politisches Selbstverständnis der Mitgliedstaaten und ihrer Bevölkerungen auf das politisch Machbare aus. Je größer und heterogener die Europäische Union ist, desto schwieriger auch der Prozeß der Gemeinschaftsbildung. Das Problem im wesentlichen auf die institutionelle Frage zu reduzieren, ist eine grobe Simplifizierung. Mit der in Amsterdam halbherzig in Angriff genommenen Ausweitung der Bereiche von Mehrheitsentscheidungen im Rat und Mitentscheidungen im

Siehe zu diesem, auf ein System "überlappender Integrationsräume und -systeme"hinauslaufendem Verständnis T. Bruha / T. Straubhaar, "EWR II": Europäischer Warteraum oder Stufenplan für Mittel- und Osteuropa?, in: R. Hasse /W. Schäfer (Hrsg.), Die ökonomischen Außenbeziehungen der EWU, 1997, S. 175-198.

So aber Vedder (oben Fn. 33), Art. 237, Rn. 1, bezüglich Anträgen auf EU-Mitgliedschaft. 
Parlament wird die erweiterte Gemeinschaft vergleichsweise nur an der Oberfläche einigermaßen funktionsfähig gehalten. Die schwierigere Aufgabe, die Bürger dieser Gemeinschaft zu einer einheitlichen europäischen "civil society" (nicht unbedingt europäisches Volk!) zusammenzuführen, kann damit nicht bewältigt werden. Im Gegenteil, sie wird zunächst einmal erschwert, da die zu überwindenden Barrieren sprachlicher, kultureller, emotionaler Art. usw. erhöht bzw. neue hinzugefügt werden.

Diese Dimension wird in der aktuellen Erweiterungsdebatte völlig unzureichend reflektiert. Um nicht mißverstanden zu werden: Man muß kein Gegner der Erweiterung der Europäischen Union, auch nicht der "großen" Erweiterung sein, wenn man auf diese Implikationen und die mit ihnen verbundenen Probleme hinweist. Der Prozeß der Vertiefung der politischen Integration schließt rechtlich zwingend die weitere Demokratisierung der Gemeinschaft ein. Dazu bedarf es nicht nur institutioneller Reformen. Es bedarf auch gewisser Voraussetzungen, die das Recht nicht selbst schaffen kann. Im vielgescholtenen Maastricht-Urteil des BVerfG findet sich der richtige Satz, daß "Demokratie, soll sie nicht lediglich formales Zurechnungsprinzip bleiben, vom Vorhandensein vorrechtlicher Voraussetzungen abhängig (ist), wie einer ständigen freien Auseinandersetzung zwischen sich begegnenden sozialen Kräften, Interessen und Ideen, in der sich auch politische Ziele klären und wandeln und aus der eine öffentliche Meinung den politischen Willen vorformt". ${ }^{84}$ Diese zutreffende Feststellung sollte bei aller berechtigten Kritik an allzusehr nationalstaatlich orientierten Homogenitätsvorstellungen des BVerfG nicht mit in Frage gestellt werden. Natürlich ist es schwieriger, im Europa der 25 oder mehr Staaten, der 22 oder mehr Nationalsprachen und der wachsenden Unterschiede und Gegensätze eine homogene Zivilgesellschaft, ein europäisches Identitätsbewußtsein, ein wirkliches "Europa der Bürger" herauszubilden. Diese Kostenseite der Erweiterung wird in der öffentlichen Diskussion zur Zeit nicht angemessen thematisiert. Es sind Abwägungen und Entscheidungen zu treffen, die aus rechtlicher Sicht nur in eine verfassungspolitischen Debatte eingebettet sein können. "Amsterdam" hat hierzu noch keine Lösungsansätze aufgezeigt. 
national community of states, their constitutional systems and their position in international law. Other aspects the Journal dealt with over the last decades are the validity of customary norms of international law for newly independent states, weighted voting in international organizations, the shape of a new international economic order, especially the right to development and the acknowledgement of resolutions of international organizations as new sources of international law, to name just as few. The article presents and considers these issues in the context of the overall developments of international law.

\section{The Expansion of the European Union: The Relevant Provisions of European Law}

\section{By Thomas Bruha and Oliver Vogt}

The European Union is facing the most extensive expansion in its history. The European Commission suggested that, in 1998, five central- and eastern European states and Cyprus should be invited to first talks on their accession to the Union. Since five more central- and eastern European states as well as Turkey, Malta and Switzerland have already applied, too, the European Union may amount to up to 35 member states after the next enlargement. Since its Essen summit in 1994, the European Union is following a double strategy in preparation for expansion, a "pre-accession" strategy for the applying states and fundamental institutional reforms for the Union itself.

In view of these developments, the provisions of the Union treaty have been emended on the 1997 Amsterdam Council of Heads of States. The article deals with the conditions of the expansion as laid down in the new Art. O European Union Treaty on the admission of new member states, featuring especially the geographical, historical and political aspects of "Europe", and comparing it to the criteria prepared on the 1993 Copenhagen summit. Emphasizing the forthcoming eastern expansion debate, the article proceeds to survey the present situation of the five candidates in view of the new criteria. It concludes with a glance at a new dimension of the debate: the development of a European "civil society". Much of this is relevant far beyond the limits of the present or future European Union.

\section{Latin American Constitutions: Between Autocracy and Democratization}

\section{By Marcelo Neves}

The constitutional development of the Latin American countries in the past three decades is characterized by a tendency shifting from autocracy in the sixties and seventies to democratization in the eighties, with growing stability in the nineties. The question arises whether 